\title{
Solving the Transshipment Problem with Fuzzy Cost Coefficients
}

\section{Elsayed Ellaimony' ${ }^{1}$ Ibrahim Ahmed ${ }^{2}$, Khaled Abdelwahed ${ }^{2}$, Rania Ahmed ${ }^{2}$ and Mohamed Khalil11,3*}

\author{
${ }^{1}$ Faculty of Engineering Helwan University, Egypt \\ ${ }^{2}$ Faculty of Industrial Education, Helwan University, Egypt \\ ${ }^{3}$ Egyptian Academy for Engineering \& Advanced Technology, Egypt \\ *Corresponding author details: Mohamed Khalil; mohamedibrahim71@yahoo.com
}

\begin{abstract}
This paper presents an optimization based mathematical modelling approach for a transshipment problem with fuzzy cost coefficients is formulated and solved as a quadratic mixed integer linear programming problem. In this paper, it is illustrated how convert the fuzzy form to crisp form an illustrative numerical example is gives to clarify the formulation and the solution.
\end{abstract}

Keywords: transportation problem; transshipment, fuzzy; quadratic programming.

\section{INTRODUCTION}

The Classical transportation problem refers to a special class of linear programming problems. In a typical problem a product is to be transported from $m$ sources to $n$ destinations and their Capacities are $a_{l}, a_{2} \ldots a_{m}$ and $b_{1}$ $b_{2}, \ldots b_{n}$ respectively. In addition, there is a penalty $C_{i j}$ associated with transporting a unit of product from source $i$ to destination $j$. This penalty may be cost or deterioration or delivery time, etc. A variable $x_{i j}$ represents the unknown quantity to be shipped from source $i$ to destination $j$. In that problem cities where goods are produced (sources) ship only to cities where goods are consumed (destinations), shipments do not take place between sources or between destinations, nor form destinations to origins. This may not always be the case. Instead of shipping directly from source to destination, it may be possible to transship, i.e. The goods shipped from some sources to some destinations via other sources and destinations where transshipment occurred at these points. This possibility may give an optimal solution with a lower total transport cost than that which given by the optimal solution of the classical transportation problem. This is done because the starting at solution of transshipment problem is the optimum in solution of the transportation problem (Usually, in the absence of the transshipment, the transportation cost goes higher). Hence the transshipment is very useful to reduce the transportation cost. Transshipment models can be used to enhance cost efficient movement of goods and improve the level of Customer satisfaction.

Transshipment problem was first introduced by Orden (1956) [3]. He gave an extension of the original Transportation problem to include the possibility of transshipment i.e., any shipping or receiving pinto is also permitted to act as an intermediate point. The Transshipment technique is used to find the shortest route from one point in a network to another.
Grag and Prakash (1985) [4] studied time minimizing transshipment problem. Osman and Ellaimony (1986) [2] introduce mathematical formulations of different multistage transportation problem with transshipments and an algorithm for solving a class of them later, dynamic transshipment problem was studied by Hererand Tzur (2001) [5]. Afterwards, Multilocation transshipment problem with Capacitated production and lost sales was studied by Ozdemir (2006) [6] Deniz T.E., Emine and Dicle in [1] use integer - programming to solve the transshipment problem of an apparel company.

Transshipment problem in the standard form is basically a linear min-cost network flow problem and for such types of optimization problems, a number of effective solutions are available since many years when the cost Coefficients and the supply and demand quantities are known exactly. However, there are cases that these Parameters may not be presented in a precise manner. For example; the unit shipping cost may vary in a time frame. The supplies and demands may be uncertain due to some uncontrollable factors.

To deal with this kind of problems Bellman and Zadeh [7] and Zadeh [8] introduce the notion of fuzziness Since the transportation problem is essentially a linear program, one straight forward idea is to apply the fuzzy Linear programming techniques to the fuzzy transportation problem. There are also studies discussing the fuzzy transportation problem. Chanas et al [9] investigate the transportation problems with fuzzy supplies and demands and solve them via parametric programming technique in terms of the Bellman Zadeh criterion. Chanas and Kuchta [10] discuss the type of transportation problems with fuzzy Cost coefficients and transform the problem to a bicriterial transportation problem with Crisp objective function. 
In this paper we introduce the mathematical Formulation of transshipment problem with fuzzy cost Coefficients and solve it as a quadratic mixed integer linear programming problem by illustrative example.

\section{MATHEMATICAL FORMULATION OF TRANSSHIPMENT PROBLEM}

In order to obtain the mathematical formulation of Transshipment problem let us assume that $m$ is the number of origins and $n$ is the number of destinations. Since in a transshipment problem, any origin or destination can ship to any other origin or destination that the origins are numbered from 1 to $m$ and the destinations from $m+1$ to $m+n$

Let $a_{i}$ is the quantities available at the origins and $b_{j}$ be the demands at the destinations and

$$
\sum_{i=1}^{m} a_{i}=\sum_{j=1}^{n} b_{j}
$$

is the coefficient of transportation cost of shipping from $i$ to $\mathrm{j}(\mathrm{i}, \mathrm{j}=1,2, \ldots, m+n \mathrm{i} \neq \mathrm{j})$ where $\mathrm{c}_{\mathrm{ij}}$. need not be the same as $c_{i j}$ and $x_{i j}(i, j=1,2, m+n i \neq j)$.

The total amount shipped from an origin must be equal to the amount it produces plus what it transships. Also, the total amount received at a destination must be equal to its demand plus what it transships. Thus, the transshipment problem takes the following form:

$\min z=\sum_{i=1}^{m+n} \sum_{\substack{j=1, j \neq i}}^{m+n} c_{i j} x_{i j}$

Subject to

$$
\begin{aligned}
& \sum_{\substack{j=1, j \neq i}}^{m+n} x_{i j-} \sum_{\substack{j=1, j \neq i}}^{m+n} x_{j i=} a_{i}, \quad i=1,2, \ldots, m ; \\
& \sum_{\substack{j=1 \\
j \neq i}}^{\substack{j \neq i \\
m+n}} x_{i j-} \sum_{\substack{j=1 \\
j \neq i}}^{\substack{j \neq i \\
m+n}} x_{j i}=b_{j}, \quad j=1,2, \ldots, m+n \text {; } \\
& x_{i j}^{j \neq i} \geq 0, \quad i, j=1,2, \ldots m+n, i \neq j
\end{aligned}
$$

The above formulation a linear programming problem, which is similar to a transportation problem but not exactly since the $\sum_{j=1}^{m+n} x_{i j}$ has negative signed the $j \neq i$

problem however may easily be converted to standard transportation problem.

let

$$
\begin{aligned}
L & =\sum_{\substack{j=1 \\
j \neq i}}^{m+n} x_{i j}=\sum_{\substack{i=1 \\
j \neq i}}^{m+n} x_{i j} \\
L & =\sum_{i=1}^{m} a_{i j}=\sum_{j=1}^{n} b_{j}
\end{aligned}
$$

Then the transshipment problem reduces to:

$\min z=\sum_{j=1}^{m+n} \sum_{j=1}^{m+n} c_{i j} x_{i j}$

Subject to

$\sum_{j=1}^{m+n} x_{i j}=a_{i}+L, \quad i=1,2, \ldots, m$ $\sum_{j=1}^{m+n} x_{i j}=L, \quad i=m+1, m+2, \ldots, m+n ;$
$\sum_{\substack{i=1 \\ m+n}}^{m+n} x_{i j}=b_{J}+L, \quad j=1,2, \ldots, n ;$
$\sum_{i=1}^{m+n} x_{i j}=L, \quad j=n+1, n+2, \ldots, n+m ;$

$\forall x_{i j} \geq 0, i=1,2, \ldots \ldots m+n ; j=1,2, \ldots . ., n+m$.

where $\mathrm{C}_{\mathrm{ij}}=0, \mathrm{i}=1,2, \ldots \mathrm{m}+\mathrm{n}$.

The above mathematical model represents a Standard Transportation problem with $(m+n)$ origins and $(m+n)$ destinations.

The mathematical models of the transshipment problem include the following:

1) The total amount shipped from an origin must be equal to the amount it produces plus what if transships. Similarly, the total amount received at a destination must be equal to its demand plus what it transships.

2) I can be interpreted as a reserve stock at each origin and destination. Since we assume that any amount of goods can be transshipped at each point, I should be large enough to take care of all transshipments.

3) The solution of the problem contains $2 m+2 n-1$ basic variables. However, $m+n$ of these variables appearing in the diagonal cells represent the remaining reserve stock and if they are omitted, we have $(m+n-1)$ basic Variables.

\section{FORMULATION OF TRANSSHIPMENT PROBLEM WITH FUZZY COST COEFFICIENTS (FCTP)}

$\min z=\sum_{i=1}^{m+n} \sum_{\substack{j=1 \\ j \neq i}}^{m+n} c_{i j} x_{i j}$

Subject to

$\sum_{j=1}^{m+n} x_{i j}=a_{i}+L, \quad i=1,2, \ldots, m$;

$\sum_{j=1}^{m+n} x_{i j}=L, \quad i=m+1, m+2, \ldots, m+n$,

$\sum_{\substack{i=1 \\ m+n}}^{m+n} x_{i j}=b_{j}+L, \quad j=1,2, \ldots, n$

$\sum_{i=1}^{m+n} x_{i j}=L, j=n+1, n+2, \ldots, n+m$,

$\forall x_{i j \geq 0} \quad, i=1,2, \ldots, m+n, j=1,2, \ldots, n+m$

where $c_{i j}=0, i=1,2, \ldots, m+n$;

The above problem is a quadratic mixed integer linear programming problem in its nature, where $\tilde{c}_{\mathrm{ij}}$ is a fuzzy cost coefficient.

Through this paper, we assume that $\tilde{c}_{\mathrm{ij}}, \mathrm{i}=1,2, \ldots, \mathrm{m}+\mathrm{n}$; $j=1,2, \ldots, m+n$ and $C_{i j}=0, i=1,2, \ldots, m+n$ are fuzzy numbers whose membership functions are:

$\mu_{\text {cij }}\left(c_{i j}\right) ; i=1,2, \ldots, m+n ; j=1,2, \ldots, m+n$.

Now, we introduce the concept of $\alpha$-level set or $\alpha$-cut of the fuzzy numbers $\tilde{c}_{\text {ij }}$ as follows:

Definition: (Dubois and Prade [11] The $\alpha$ level set of the fuzzy numbers $\tilde{c}_{\mathrm{ij}}$ is defined as the ordinary set $\mathrm{L}_{\alpha}\left(\tilde{c}_{\mathrm{ij}}\right)$ for 
which the degree of their membership function exceeds the level $\alpha \in[0,1]$.

$\mathrm{L}_{\alpha}\left(\tilde{c}_{\mathrm{ij}}\right)=\left\{\tilde{c}_{\mathrm{ij}} / \mu \tilde{c}_{\mathrm{ij}}\left(\tilde{c}_{\mathrm{ij}}\right) \geq \alpha(\mathrm{i}=1,2, . . \mathrm{m}+\mathrm{n} ; \mathrm{j}=1,2, . ., \mathrm{m}+\mathrm{n}\}\right.$

For a certain degree $\alpha$ the transshipment Problem with fuzzy Cost Coefficients can be understood as the following non fuzzy $\alpha$-transshipment quadratic mixed integer linear programming problem ( $\alpha$-Fuzzy Cost transshipment problem,) $(\alpha$-FCTP)

\section{$(\alpha$-FCTP):}

$\min z=\sum_{i=1}^{m+n} \sum_{\substack{j=1 \\ j \neq i}}^{m+n} c_{i j} x_{i j}$

Subject to

$$
\begin{aligned}
& \sum_{j=1}^{m+n} x_{i j}=a_{i}+L, \quad i=1,2, \ldots, m ; \\
& \sum_{\substack{m+n \\
m+n}}^{m+n} x_{i j}=L, \quad i=m+1, m+2, \ldots, m+n ; \\
& \sum_{\substack{i=1 \\
m+n}}^{m+n} x_{i j}=b_{j}+L, \quad j=1,2, \ldots, n ; \\
& \sum_{i=1}^{m+n} x_{i j}=L, \quad i=n+1, n+2, \ldots, n+m ;
\end{aligned}
$$

$x_{i j} \geq 0$ and inteeger for all $i, j$;

$\mathrm{C}_{\mathrm{ij}} \in \mathrm{L} \alpha\left(\tilde{c}_{\mathrm{ij}}\right)$;

$c_{i j}=0, i=1,2, \ldots, m+n$

In the $(\alpha-F C T P)$, the parameters $c_{i j}(i=1,2, \ldots, m+n)$ are treated and decision variables rather than constants.

Based on the definition of $\alpha$ level set of fuzzy numbers, the $\alpha$-FCTP will be treated using quadratic mixed integer linear programming problem with the following formulation:

$\min z=\sum_{i=1}^{m+n} \sum_{\substack{j=1 \\ j \neq i}}^{m+n} c_{i j} x_{i j}$

Subject to

$$
\begin{aligned}
& \sum_{j=1}^{m+n} x_{i j}=a_{i}+L, \quad i=1,2, \ldots, m ; \\
& \sum_{j=1}^{m+n} x_{i j}=L, \quad i=m+1, m+2, \ldots, m+n, \\
& \sum_{\substack{i=1 \\
m+n}}^{m+n} x_{i j}=b_{j}+L, \quad j=1,2, \ldots, n \\
& \sum_{i=1}^{m+n} x_{i j}=L, \quad j=n+1, n+2, \ldots, n+m,
\end{aligned}
$$

$x_{i j} \geq 0$ and inteeger for all $i, j$;

$h_{i j} \leq c_{i j} \leq H_{i j}, \mathrm{i}=1,2, . ., \mathrm{m}+\mathrm{n} ; \mathrm{j}=1,2, . ., \mathrm{m}+\mathrm{n}$;

$c_{i j}=0, \quad i=1,2, \ldots, m+n$

\section{Where:}

$h_{i j}, H_{i j}$ are lower and upper boundson $c_{i j}$ respectively Then the constraints al the problem takes the following form:

$\sum_{j=1}^{m+n} x_{i j}=a_{i}+L, \quad i=1,2, \ldots, m$; $\sum_{j=1}^{m+n} x_{i j}=L, \quad i=m+1, m+2, \ldots, m+n$,

$\sum_{i=1}^{m+n} x_{i j}=b_{j}+L, \quad j=1,2, \ldots, n$

$\sum_{i=1}^{n+m} x_{i j}=L, i=n+1, n+2, \ldots, n+m$

$x_{i j} \geq 0$ and ineeger for all $i, j$;

$c_{i j} \leq H_{i j}$

$c_{i j} \leq H_{i j}, \mathrm{i}=1,2, . ., \mathrm{m}, \mathrm{n}, \mathrm{j}=1,2, . ., \mathrm{m}+\mathrm{n}$;

$c_{i j}=0, \mathrm{i}=1,2, . ., \mathrm{m}, \mathrm{n}$;

\section{ILLUSTRATIVE EXAMPLE}

To illustrate the fuzzy approach of Transshipment problem

\begin{tabular}{|c|c|c|c|c|}
\hline$i^{j}$ & D1 & D2 & S1 & S2 \\
\hline S1 & $\widetilde{C_{11}}$ & $\widetilde{C_{12}}$ & $\widetilde{C_{13}}=0$ & $\widetilde{C_{14}}$ \\
\hline S2 & $\widetilde{C_{21}}$ & $\widetilde{C_{22}}$ & $\widetilde{C_{23}}$ & $\widetilde{C_{24}}=0$ \\
\hline D1 & $\widetilde{C_{31}}=0$ & $\widetilde{C_{32}}$ & $\widetilde{C_{33}}$ & $\widetilde{C_{34}}$ \\
\hline D2 & $\widetilde{C_{41}}$ & $\widetilde{C_{42}}=0$ & $\widetilde{C_{43}}$ & $\widetilde{C_{44}}$ \\
\hline
\end{tabular}
with fuzzy cost coefficients let us consider the following example:

The problem is Transshipment problem with a two origins $(1,2)$ with availabilities $(8 \mathrm{~T}, 7 \mathrm{~T})$ and two destinations $(3,4)$ With requirements(9T, 6T) respectively and fuzzy cost coefficients $\tilde{c}_{\mathrm{ij}}$, $\mathrm{i}=1,2, . .4, j=1,2, . .4 \quad \mathrm{c}_{\mathrm{ij}}=0 ; \mathrm{i}=\mathrm{j}=1,2, . .4$ as show in the following TABLE 1 :

TABLE 1: Represents the Transshipment Problem Table

$L=\sum_{i=1}^{3} a_{i}=\sum_{j=1}^{3} b_{j}=15 T$

(FCTP):

Min Z

$=\tilde{c}_{11} \mathrm{X}_{11}+\tilde{c}_{12} \mathrm{X}_{12}+\tilde{c}_{13} \mathrm{X}_{13}+\tilde{c}_{14} \mathrm{X}_{14}+\tilde{c}_{21} \mathrm{X}_{21}+\tilde{c}_{22} \mathrm{X}_{22}+\tilde{c}_{23} \mathrm{X}_{23}+\tilde{c}_{24} \mathrm{X}_{24}$ $+\tilde{c}_{31} \mathrm{X}_{31}+\tilde{c}_{32} \mathrm{X}_{32}+\tilde{c}_{33} \mathrm{X}_{33}+\tilde{c}_{34} \mathrm{X}_{34}+\tilde{c}_{41} \mathrm{X}_{41}+\tilde{c}_{42} \mathrm{X}_{42}+\tilde{c}_{43} \mathrm{X}_{43}+\tilde{c}_{44} \mathrm{X}_{44}$

Subject to:

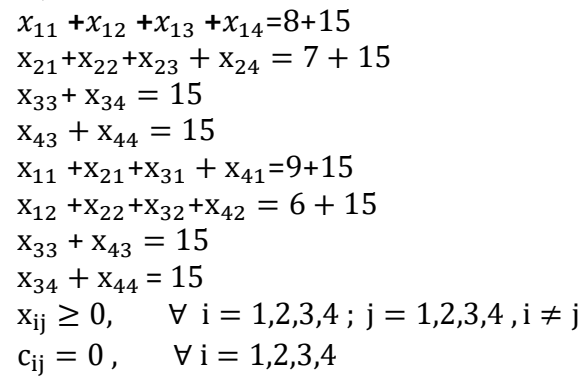

Assume that the membership function corresponding to the fuzzy numbers

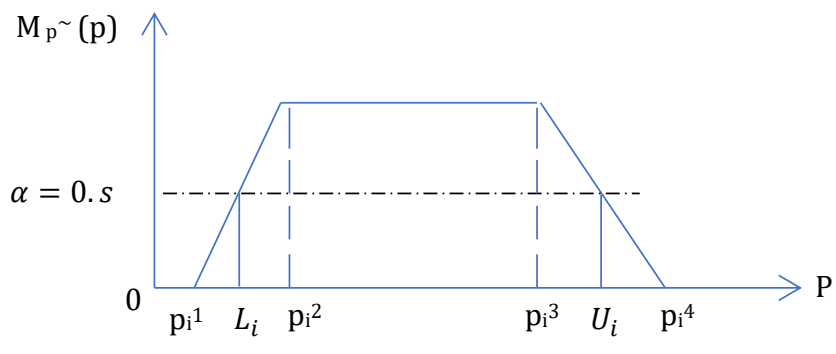


MEMBERSHIP FUNCTION OF A FUZZY NUMBER $\tilde{\boldsymbol{p}}$. THE ABOVE FIGURE ILLUSTRATES THE GRAPH OF A POSSIBLE SHAPE OF FUZZY NUMBER $\widetilde{p}_{i}$

$$
\mu \tilde{c}_{\mathrm{ij}}\left(\mathrm{c}_{\mathrm{ij}}\right)\left\{\begin{array}{cl}
\mathbf{0} & \leq P_{j}^{1} \\
\frac{\boldsymbol{c}_{\boldsymbol{i j}}-\boldsymbol{p}_{\boldsymbol{j}}^{\mathbf{1}}}{\boldsymbol{p}_{\boldsymbol{j}}^{\mathbf{2}}-\boldsymbol{p}_{\boldsymbol{j}}^{\mathbf{1}}} & P_{j}^{1} \leq c_{i j} \leq P_{j}^{2} \\
\mathbf{1} & P_{j}^{2} \leq c_{i j} \leq P_{j}^{3} \\
\frac{\boldsymbol{c}_{\boldsymbol{i j}}-\boldsymbol{p}_{\boldsymbol{j}}^{\mathbf{3}}}{\boldsymbol{p}_{\mathbf{j}}^{\mathbf{4}}-\boldsymbol{p}_{\boldsymbol{j}}^{\mathbf{3}}} & P_{j}^{3} \leq c_{i j} \leq P_{j}^{4} \\
\mathbf{0} & P_{j}^{4} \leq c_{i j} \leq \infty
\end{array}\right.
$$

For $\alpha$-cut $=0.5$

The following Table 2 illustrate values of upper and lower bounds of the Cost coefficients $\left(\boldsymbol{c}_{\boldsymbol{i} j}\right)$

TABLE 2: The Upper \& Lower Boundary of the Cost of Objective Function

\begin{tabular}{|c|c|c|c|c|c|c|}
\hline $\mathbf{C}_{\mathbf{i j}}$ & $\boldsymbol{P}_{\boldsymbol{i}}^{\mathbf{1}}$ & $\boldsymbol{P}_{\boldsymbol{i}}^{\mathbf{2}}$ & $\boldsymbol{P}_{\boldsymbol{i}}^{\mathbf{3}}$ & $\boldsymbol{P}_{\boldsymbol{i}}^{\mathbf{4}}$ & $\mathbf{L}_{\mathbf{i j}}$ & $\mathbf{U}_{\mathbf{i j}}$ \\
\hline $\mathrm{C}_{11}$ & 4 & 7 & 8 & 9 & 5.5 & 8.5 \\
\hline $\mathrm{C}_{12}$ & 2 & 5 & 6 & 8 & 3.5 & 7 \\
\hline $\mathrm{C}_{13}$ & 0 & 0 & 0 & 0 & 0 & 0 \\
\hline $\mathrm{C}_{14}$ & 2 & 4 & 7 & 9 & 3 & 8 \\
\hline $\mathrm{C}_{21}$ & 2 & 4 & 5 & 7 & 3 & 6 \\
\hline $\mathrm{C}_{22}$ & 1 & 2 & 4 & 6 & 1.5 & 5 \\
\hline $\mathrm{C}_{23}$ & 2 & 3 & 4 & 6 & 2.5 & 5 \\
\hline $\mathrm{C}_{24}$ & 0 & 0 & 0 & 0 & 0 & 0 \\
\hline $\mathrm{C}_{31}$ & 0 & 0 & 0 & 0 & 0 & 0 \\
\hline $\mathrm{C}_{32}$ & 1 & 3 & 4 & 6 & 2 & 5 \\
\hline $\mathrm{C}_{33}$ & 1 & 2 & 4 & 6 & 1.5 & 5 \\
\hline $\mathrm{C}_{34}$ & 3 & 4 & 5 & 7 & 3.5 & 6 \\
\hline $\mathrm{C}_{41}$ & 1 & 3 & 5 & 7 & 2 & 6 \\
\hline $\mathrm{C}_{42}$ & 0 & 0 & 0 & 0 & 0 & 0 \\
\hline $\mathrm{C}_{43}$ & 2 & 4 & 5 & 6 & 3 & 5.5 \\
\hline $\mathrm{C}_{44}$ & 2 & 3 & 4 & 6 & 2.5 & 5 \\
\hline
\end{tabular}

Crisp Form of the problem:,

\section{Min $\mathrm{Z}=$}

$c_{11} x_{11}+c_{12+} x_{12+} c_{13} x_{13}+c_{14} x_{14}+c_{21} x_{21}+c_{22} x_{22}+$

$c_{23} x_{23}+c_{24} x_{24}+c_{31} x_{31}+c_{32} x_{32}+c_{33} x_{33}+$

$c_{34} x_{34}+c_{41} x_{41}+c_{42} x_{42}+c_{43} x_{43}+c_{44} x_{44}$

Subject to:

$x_{11}+x_{12}+x_{13}+x_{14}=23$

$\mathrm{x}_{21}+\mathrm{x}_{22}+\mathrm{x}_{23}+\mathrm{x}_{24}=22$

$\mathrm{x}_{33}+\mathrm{x}_{34}=15$

$\mathrm{x}_{43}+\mathrm{x}_{44}=15$

$\mathrm{x}_{11}+\mathrm{x}_{21}+\mathrm{x}_{31}+\mathrm{x}_{41}=24$

$\mathrm{x}_{12}+\mathrm{x}_{22}+\mathrm{x}_{32}+\mathrm{x}_{42}=21$

$\mathrm{x}_{33}+\mathrm{x}_{43}=15$

$\mathrm{x}_{34}+\mathrm{x}_{44}=15$

$\mathrm{x}_{\mathrm{ij}} \geq 0, \quad \forall \mathrm{i}=1,2,3,4 ; \mathrm{j}=1,2,3,4, \mathrm{i} \neq \mathrm{j}$

$\mathrm{c}_{\mathrm{ij}}=0, \quad \forall \mathrm{i}=1,2,3,4$

$(\mathrm{L})_{\mathrm{ij}} \leq \mathrm{C}_{\mathrm{ij}} \leq(\mathrm{U})_{\mathrm{ij}}$

$x_{i j} \geq 0$ and integers for $\mathrm{i}=1,2,3,4 ; \mathrm{j}=1,2,3,4$;

The TABLE 2 gives the lower $\left(\mathrm{L}_{\mathrm{ij}}\right)$ and upper $\left(\mathrm{U}_{\mathrm{ij}}\right)$ bounds at the Cost Coefficients $c_{i j}$ from the membership function. The optimal mixed - integer Linear Programing solution for the transshipment problem with fuzzy Cost Coefficients in the illustrated example to given as following by lingo software program.

\section{OPTIMAL DISTRIBUTION POLICY FOR THE GOODS}

Objective value:

60.00000

Infeasibilities:

0.000000

Total solver iterations:

7

Elapsed runtime seconds: $\quad 0.12$

Model Class:

QP

Total variables:

28

Nonlinear variables: $\quad 24$

Integer variables:

Total constraints:

0

$\begin{array}{lcc}\text { Variable } & \text { Value } & \text { Reduced Cost } \\ \mathrm{X} 11 & 23.00000 & 0.000000 \\ \mathrm{C} 12 & 3.000000 & 0.000000 \\ \mathrm{C} 13 & 5.500000 & 0.000000 \\ \mathrm{C} 14 & 3.500000 & 0.000000 \\ \mathrm{C} 21 & 2.500000 & 0.000000 \\ \mathrm{X} 21 & 1.000000 & 0.000000 \\ \mathrm{X} 22 & 21.00000 & 0.000000 \\ \mathrm{C} 23 & 3.000000 & 0.000000 \\ \mathrm{C} 24 & 1.500000 & 0.000000 \\ \mathrm{C} 41 & 2.000000 & 0.000000 \\ \mathrm{C} 42 & 3.000000 & 0.000000 \\ \mathrm{C} 43 & 2.500000 & 0.000000 \\ \mathrm{X} 44 & 15.00000 & 0.000000 \\ \mathrm{C} 31 & 1.500000 & 0.000000 \\ \mathrm{C} 32 & 3.500000 & 0.000000 \\ \mathrm{X} 33 & 15.00000 & 0.000000 \\ \mathrm{C} 34 & 2.000000 & 0.000000\end{array}$

\section{CONCLUSION}

In this paper, we have presented a mathematical formulation for transshipment problem with fuzzy cost coefficient (FCTP) and solved as quadratic mixed integer linear programming problem. In our opinion, we feel that, there are many other points of research and should be studied: First; an algorithm is needed for solving large scale transshipment problems having fuzzy parameters in the right hand side of the constraints, and second; an algorithm is needed for solving large scale transshipment problems having fuzzy cost coefficients.

\section{REFERENCES}

[1] Deniz T.E., Emine z. y. and DicleY.S. "Supply chain Management in Apparel Industry: A Transshipment problem with Time constraints." Tektilve Konfekslyon (2/2011) pp. 176-181.

[2] Osman M.S.A. and Ellaimony E.E.M. "An Algorithm for Solving large scale Transshipment Problems" Indian Journal of Technology vol.24 (June 1986), pp.294-392.

[3] Orden, A. "Transshipment problems" Management Science, (3), (1956), 276-285.

[4] Grag, R and Prakash, S." Time Minimizing Transshipment Problem" Indian Journal of Pure and Applied Mathematics, Vol. 16 (5) (1985): 449-460.

[5] Henery and Tzur, M. "The Dynamic Transshipment Problem". Naval Research Logistics Quaterely, 48 (2001): 386 -408.

[6] Ozdemir, D. Y ücesan, E. and Herer, t." Multilocation Transshipment Problem with Capacitated Production and Lost sales. Proceedings of the 2006 Winter Simulation Conterence, Pages 1470 -1476. 
[7] Bellman, R. Zadeh L. A," Decision making in a fuzzy Environment", Management sci 17(b) (1970) 141-164.

[8] Zadeb. L.A., "Fuzzy Sets AS A Basis for A Theory of Possibility". Fuzzy Sets and Systems, 1(1978) 3-28.

[9] Chanass. Kolodziejczy K W. and Machaj A., "A Fuzzy approach to transportation problem", Fuzzy Sets and Systems 13 (1984).
[10] Chanas S., Kuchta D., "A concept of the optimal solution of the Transportation problem with Fuzzy Cost Coefficients", Fuzzy Sets and Systems 82(1996) 299-309.

[11] D. Dubois, A. Prade, "Fuzzy sets and systems. Theory and applications", Academic Press, New York, 1980. 